\title{
Building a Knowledge to Action Program in Stroke Rehabilitation
}

\author{
Shannon Janzen, Amanda McIntyre, Marina Richardson, Eileen Britt, \\ Robert Teasell
}

\begin{abstract}
The knowledge to action (KTA) process proposed by Graham et al (2006) is a framework to facilitate the development and application of research evidence into clinical practice. The KTA process consists of the knowledge creation cycle and the action cycle. The Evidence Based Review of Stroke Rehabilitation is a foundational part of the knowledge creation cycle and has helped guide the development of best practice recommendations in stroke. The Rehabilitation Knowledge to Action Project is an audit-feedback process for the clinical implementation of best practice guidelines, which follows the action cycle. The objective of this review was to: (1) contextualize the Evidence Based Review of Stroke Rehabilitation and Rehabilitation Knowledge to Action Project within the KTA model and (2) show how this process led to improved evidence-based practice in stroke rehabilitation. Through this process, a single centre was able to change clinical practice and promote a culture that supports the use of evidence-based practices in stroke rehabilitation.
\end{abstract}

RÉSUMÉ: Construire un programme de passage du savoir à l'action dans la réadaptation de l'accident vasculaire cérébral. Le processus de passage du savoir à l'action (PSA) proposé par Graham et al (2006) constitue un cadre dont le but est de faciliter l'élaboration et l'application de données de recherche en pratique clinique. Le processus (PSA) comprend le cycle de création du savoir et le cycle de l'action. Le Evidence-Based Review of Stroke Rehabilitation (EBRSR) est une partie fondamentale du cycle de la création du savoir et a aidé à guider l'élaboration des recommandations de pratiques exemplaires pour l'accident vasculaire cérébral (AVC). Le Rehabilitation Knowledge to Action Project (REKAP) est un processus de vérification et de rétroaction portant sur la mise en application des lignes directrices sur les pratiques exemplaires et suit le cycle d'action. L'objectif de cette revue était de contextualiser l'EBRSR et le REKAP dans le cadre du modèle PSA et de montrer comment ce processus a mené à une amélioration de la pratique basée sur des données probantes dans la réadaptation de l'AVC. Grâce à ce processus, un centre a pu modifier la pratique clinique et promouvoir une culture qui soutient l'utilisation de pratiques basées sur des données probantes dans la réadaptation de l'AVC.

Keywords: stroke, rehabilitation, practice guidelines, knowledge translation, evidence-based, adherence

doi:10.1017/cjn.2016.258

Can J Neurol Sci. 2016; 43: 619-625

Stroke is one of the leading causes of death and disability in Canada. $^{1}$ Approximately two-thirds of stroke survivors experience a loss of function in physical, cognitive, or communication skills. ${ }^{2}$ Consequently, it is imperative that individuals with stroke receive care that promotes optimal recovery. The integration of clinical expertise with high-quality research evidence is the cornerstone of evidence-based medicine. ${ }^{3}$ A health care system that uses the best research evidence optimizes patient outcomes; however, only $55 \%$ to $67 \%$ of patients reportedly receive care consistent with best evidence. ${ }^{4,5}$

With the consequences of stroke well-known, a substantial body of published research evidence pertaining to stroke interventions exists. Despite the volume of stroke research, the process of translating the knowledge obtained from such studies into clinical practice has lagged, with some estimates reaching 17 years for implementation to take place, if at all. ${ }^{6}$ A consistent finding in the literature is the lack of uptake of clinical and health services research into both practice and policy. ${ }^{7}$ However, the translation of research evidence into patient care settings is challenging.

Many models for knowledge translation exist, including the knowledge to action (KTA) cycle developed by Graham et $\mathrm{al}^{8}$ (Figure 1). This framework proposes a dynamic and iterative process that consists of two interacting processes: (1) the knowledge creation cycle and (2) the action cycle. The knowledge creation cycle consists of three phases and involves distilling knowledge from its most basic form to create a collection of synthesized, appraised, and user-friendly products catered to the needs of researchers and clinicians alike. The Evidence Based Review of Stroke Rehabilitation (EBRSR; www.ebrsr.com) ${ }^{9}$ serves as one example of knowledge synthesis and is a resource designed to inform evidence-based practice. The EBRSR, now in its 16th edition, is a comprehensive review of the evidence for stroke rehabilitation that covers the continuum of care. Another important part of the knowledge creation cycle is knowledge

From the Lawson Health Research Institute, Parkwood Institute, London, Ontario, Canada (SJ, AM, RT); University Hospital, London Health Sciences Centre, London, Ontario, Canada (AM); Canadian Agency for Drugs and Technologies in Health, Ottawa, Ontario, Canada (MR); Parkwood Institute, St. Josephs Health Care London, London, Ontario, Canada (EB, RT); Department of Physical Medicine and Rehabilitation,

Schulich School of Medicine and Dentistry, Western University, London, Ontario, Canada (RT).

Received August 14, 2015. Final Revisions Submitted January 5, 2016. Date of ACCEPTANCE FEBRUary 16, 2016.

Correspondence to: Shannon Janzen, Lawson Health Research Institute, Parkwood Institute, Main Building (B3-123b), 550 Wellington Road, London, ON, N6C 0A7. Email: Shannon.Janzen@sjhc.london.on.ca 


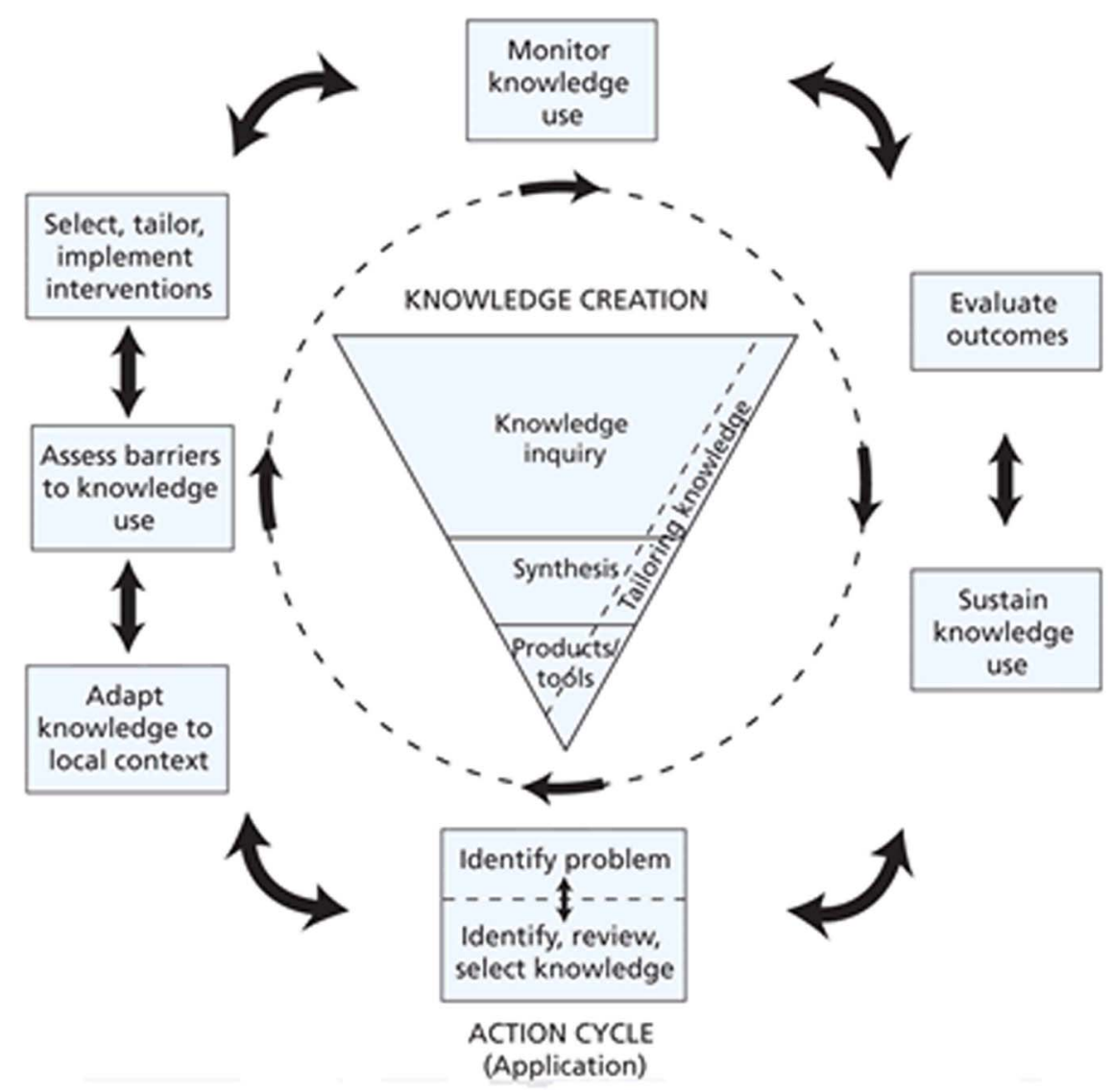

Figure 1: The knowledge to action process (Reprinted from Graham et al. 2006, copyright (C) 2006, with permission from John Wiley \& Sons, Inc.).

tools, such as clinical practice guidelines (CGPs; e.g. the Canadian Best Practice Recommendations for Stroke Care).

The action cycle is the process of moving knowledge into practice. The seven components of the action cycle were derived from a review of more than 60 theories and frameworks for planned action, which were intended to guide and promote change within a social system. ${ }^{8}$ The stages include: (1) identifying a problem, (2) adapting knowledge, (3) assessing barriers, (4) implementing, (5) monitoring, (6) evaluating, and (7) sustaining. ${ }^{8}$ Researchers have used this framework to guide knowledge translation initiatives within stroke rehabilitation for unilateral spatial neglect ${ }^{10}$ and for speech-language therapy. ${ }^{11}$

In an effort to investigate and improve CPG adherence on a stroke inpatient rehabilitation unit in Ontario, Canada, the Rehabilitation Knowledge to Action Project (REKAP) was developed. Guided by the KTA model by Graham et al, ${ }^{8}$ this project sought to improve guideline adherence in care areas where clinical practice gaps were identified. This article will discuss the utility of the KTA model using two examples, the EBRSR (knowledge creation) and REKAP (action cycle), in terms of methodology and clinical implications.

\section{Knowledge Creation Cycle}

The EBRSR is a comprehensive database that synthesizes stroke rehabilitation research. The EBRSR is designed to help frontline clinicians, policy makers, researchers, and educators manage and take advantage of the growing wealth of stroke rehabilitation evidence. The EBRSR can be accessed online for free and is updated annually.

\section{KNOWLEDGE INQUIRY}

Knowledge inquiry is often referred to as "first-generation knowledge" and represents a collection of evidence in its most basic form. ${ }^{8}$ This knowledge encompasses primary research studies and provides the foundation for the subsequent steps of knowledge synthesis and the development of knowledge products. For randomized controlled trials (RCTs) alone, Glasziou and Haynes ${ }^{12}$ estimated that approximately 55 new studies are indexed in the Cochrane central database per day. There has also been an unprecedented acceleration in the amount of stroke rehabilitation research being conducted, with the number of RCTs in stroke rehabilitation increasing threefold over the past decade. More specifically, 1063 stroke rehabilitation RCTs were published from 1970 to 2012, 370 of which were published in the most recent 5 years. ${ }^{13}$ Given the amount of evidence available and the pace at which new research is produced, keeping up to date on the latest research findings is challenging.

The Canadian Institute of Health Research ${ }^{14}$ has acknowledged the importance of systematic reviews in developing evidence-based practice and planning clinical trials. These reviews and the effective dissemination of that knowledge are critical to effective decision making and improving health 
outcomes in a way that is both financially sustainable and costeffective. This has been echoed in the Drummond Report ${ }^{15}$ to the Ontario government, which emphasized the need for "evidence-based policy" and the need for "research-based clinical guidelines" in determining future health care priorities and which services will be funded. The bridge between primary research studies and the final products that lead to such policies and system changes are resources such as the EBRSR.

\section{KNOWLEDGE SYNTHESIS}

The synthesis of research is a complex process but important for tailoring knowledge within the knowledge creation cycle. Individual trials are often single site, involve heterogeneous populations, and are of variable methodological quality, making the generalizability of findings difficult. Further, many studies are designed without considering existing research, ${ }^{16}$ and many researchers do not discuss their results within the context of other studies. ${ }^{17}$ The value of primary research studies are often better elucidated when they are combined and reviewed within the realm of existing literature.

The EBRSR aggregates and synthesizes the evidence that is identified during the knowledge inquiry stage. The EBRSR identifies relevant articles within key areas of stroke rehabilitation through systematic literature searches in multiple scientific databases. Articles are then organized according to the type of intervention assessed and the type of study design used. RCTs are summarized in the form of evidence tables and assessed for methodological quality using the Physiotherapy Evidence Database tool. ${ }^{18}$ All studies assessing a particular intervention are then considered as a whole and a level of evidence is assigned according to the results, the number of studies assessed, and their associated Physiotherapy Evidence Database scores. The EBRSR, and its methodology, have attempted to overcome some of the limitations of other reviews ${ }^{19}$ by ensuring systemwide, regularly updated, and clinically useable information in a readily accessible form.

The 16th edition of the EBRSR contained the results of 1431 RCTs, more than 1200 of which evaluated various therapies, technologies, models of care, or medications used in stroke rehabilitation; the remaining RCTs dealt with the secondary prevention of stroke. ${ }^{9}$ Together with the results of more than 2500 non-RCTS, the research has been synthesized into approximately 500 conclusion statements/levels of evidence.

\section{Knowledge Products/Tools}

The development of knowledge products and/or tools is the final phase of the knowledge creation cycle. The EBRSR provides "knowledge-transfer ready" information in the form of key points, summaries, and levels of evidence. The website also contains a clinician's handbook and education modules that are tailored to clinicians at various stages of training. The availability of such knowledge products/tools has affected how stroke rehabilitation is organized and implemented in Canada. ${ }^{20}$

The EBRSR has aided in identifying the strength of the evidence for stroke rehabilitation, revealing an extensive and rich research database with which to inform clinical and policy decisions. The information contained in the reviews can also be used to identify areas in need of further research and as a clinical decision-making tool. For example, the EBRSR has contributed to basic standards for stroke rehabilitation care ${ }^{21}$ and best practice recommendations in Canada, ${ }^{22}$ among other guidelines. ${ }^{23-25}$

\section{ACTION CYCLE: REKAP}

Guided by the Graham et $\mathrm{al}^{8}$ action cycle, REKAP involved examining a number of clinical processes in stroke rehabilitation, comparing them to best practice guideline recommendations and where appropriate, attempting to increase guideline adherence. REKAP took place on a stroke inpatient rehabilitation unit within a freestanding rehabilitation hospital in Southwestern Ontario, Canada. The 26-bed unit serves individuals with stroke or neurological conditions that require comprehensive and intensive rehabilitation services by an interdisciplinary team. This section describes the methodology used for the project and provides real-life examples of knowledge translation efforts. For this article, elements of REKAP have been placed into a stage of the action cycle for which they were deemed best suited; however, the cycle is a fluid process with continuous overlap between stages.

\section{Identify Problem}

The action cycle begins with identifying a problem (the gap between what is done and what should be done) and acquiring knowledge that may be useful in addressing the problem. Stroke CPGs have become an important tool for standardization of care by providing direction on the parameters of care. ${ }^{24}$ Studies have shown a relationship between adherence to prescribed guidelines and improved patient outcomes ${ }^{26-28}$; however, successful adherence to CPGs is dependent on active implementation strategies, ${ }^{29}$ although they are typically disseminated using passive methods. ${ }^{30}$ Regardless, compliance with best practices is important in providing optimal care.

To identify gaps between best practices and actual practices on the rehabilitation unit, the 2010 Canadian Best Practice Recommendations for Stroke Care ${ }^{24}$ were reviewed; only recommendations that were written in an objective and measurable way were selected. From these, areas that were felt to be pertinent to stroke rehabilitation were chosen. Based on the selected recommendations, an abstraction form was developed and a retrospective audit of inpatient charts was conducted. For many recommendations, adherence was straightforward and could be assessed using "yes" or "no" responses (e.g. was a depression screen completed) or by recording assessment scores (e.g. scores for the Montreal Cognitive Assessment). For other items, the abstraction was individually tailored. For example, the evaluation of hypertension management included the extraction of all documented blood pressure (BP) readings and changes to antihypertensive medications for each day a patient was present on the unit. Each medical chart was reviewed in its entirety by two independent and trained abstractors. Results from each abstractor were compared; conflicting results were resolved by the third abstractor. Our audit strategy was in line with the requirements of other health care institutions; if a task was not documented, it was assumed not to be completed. ${ }^{31}$ To measure adherence in certain areas, there was a need to obtain data from existing hospital databases. For example, therapy-intensity data were gathered from the Infomed Development Corporation's software, Promiso (Comox, British Columbia, Canada), a system used to document amount of 
individual therapy time spent with patients by various clinicians and associated aides.

The initial audit examined multiple aspects of stroke rehabilitation including the assessment and management of depression, ${ }^{32}$ cognitive screening and assessment, ${ }^{33}$ bladder management, intensity of therapy, ${ }^{34}$ benzodiazepine use ${ }^{35}$ and the management of hypertension. These will be used as examples to highlight various stages of the action cycle. Abstracted data were entered into a database and served as the baseline level of adherence. Although the guidelines do state what should be done in clinical practice, $100 \%$ adherence to a guideline may not be feasible because of exceptional cases or resources. Therefore, acceptable minimums should be determined based on clinical judgment; these standards should be used as an acceptable level of adherence. For example, a minimal level of adherence was established for hypertension management. The number of days that BP was not recorded and the number of patients who experienced hypertension (BP $\geq 140 / 90 \mathrm{mmHg}$ ) were calculated. It was determined that if a patient remained hypertensive for 3 consecutive days, the third and all subsequent days without a medication change or indication that the patients medications were reviewed were considered "untreated days." When adherence to a recommendation was low, it was deemed a "care gap" or a "knowledge-practice gap," thereby identifying problem areas.

\section{Adapt Knowledge}

Adapting knowledge is a process that involves making decisions with the current clinical setting in mind regarding the value, utility, and appropriateness of the findings. ${ }^{8}$ The knowledge-practice gaps identified in the initial audit were discussed with the leader of the stroke rehabilitation unit, the stroke council, and the clinicians on the unit (i.e. physiatrists, occupational therapists, physiotherapists, speech language pathologists, nurses, residents, and dieticians). In our setting, the research and rehabilitation teams collaboratively reviewed the audit findings to prioritize the knowledge-practice gaps. To do so, the team considered: (1) the impact the guideline had on patients and their caregivers, (2) the value of the guideline itself including supporting scientific evidence, and (3) the ability of the team to increase adherence within the current system given its inherent limitations.

For each knowledge-practice gap, a multidisciplinary working group was established. Working groups consisted of a stroke coordinator, researchers, and other clinicians depending on the guideline of interest. Specific clinicians, selected based on the knowledge translation (KT) strategy, acted as knowledge brokers. Knowledge brokers are invaluable to KT strategies because they often act as the bridge between researchers and clinicians and are useful in dissolving tension and encouraging timely uptake of scientific evidence. ${ }^{36}$

\section{ASSESS BARRIERS}

Barriers could be related to the knowledge that is being implemented, the knowledge users themselves, or the setting. ${ }^{8}$ Cabana et $\mathrm{al}^{37}$ identified 293 barriers to physician guideline adherence. Given that barriers have been found to vary across centres $^{37}$ and clinical disciplines, ${ }^{38}$ it was imperative that the perceived barriers for each care gap were identified before a strategy was developed. REKAP used, most commonly, informal discussions with the clinical team to identify barriers to guideline adherence. Discussions included the relevant clinicians, members of the research team, and administrative personnel. The researcher facilitating the discussions used prompts to ensure that both barriers and facilitators pertaining to all aspects of care were considered. The prompts were broad categories of types of barriers (e.g. administrative, clinical, financial, resources, documentation, environmental) and were developed based on relevant literature. For some care gaps, such as the assessment and management of depression, ${ }^{32}$ staff questionnaires were also used to assess guideline awareness and personal opinions regarding the importance and feasibility of depression screening.

Common barriers noted in the health care literature include lack of time, staffing issues, training/education, therapy selection, and prioritization, equipment, and team dynamics. ${ }^{11,38-40}$ These barriers were identified within the REKAP study as well. In addition, there was found to be a lack of awareness that stroke guidelines even existed during REKAP ${ }^{32}$ and incongruences between what the guideline suggested versus what was done in practice based on clinical and personal opinion. Finally, charting practices were said to not be an accurate reflection of what had been done on the unit.

Although barriers can stem from the level of an individual practitioner, other barriers may be a result of systemic pitfalls. In discussing potential reasons behind low comprehensive cognitive assessment rates after stroke, McClure et $\mathrm{al}^{33}$ suggested that factors related to poor guideline adherence may include limitations of screening tools themselves, lack of necessary resources, and the lack of evidence-based interventions available for patients with cognitive impairment. From a research and management perspective, certain guidelines were found to be too vague in their wording to be evaluated; consequently, there was less accountability for these types of guidelines.

\section{IMPLEMENTATION}

This phase of the action cycle includes both the planning and execution of a strategy to facilitate knowledge uptake. The joint collaboration between the research and the rehabilitation team allowed strategies to be created and implemented without sacrificing the routine responsibilities on the unit. As noted previously, multidisciplinary working groups were established and were key to overcoming existing barriers; these groups were essential in ensuring that the strategies were clinically relevant, adhered to best practices, aligned with appropriate professional accrediting bodies, and were feasible to implement on the unit (e.g. with existing staff, resources, equipment). The interventions were multifaceted and included educational components (e.g. information sessions, case studies), alterations to documentation practices, trial periods, active reminders, and real-time feedback. Multifaceted protocols have been shown to be most effective, ${ }^{41}$ and leadership has been shown to be the main predictor of how quickly a strategy permeates through an organization. ${ }^{42}$ Our intervention protocols were facilitated by strong management involvement, open communication, and direct accountability, which facilitated the successful uptake of the knowledge translation strategies.

Implementation strategies were unique for each evidence-care gap identified. To illustrate one specific example, the management of hypertension will be discussed. Two senior Registered Nurses acted as the champions of change. The nurses offered their clinical expertise and also acted as the liaison between the 
working group and nursing staff to develop "buy in" and ensure fluid communication throughout the process. Using nursing champions helped to facilitate nursing staff to change practices and adopt evidence-based recommendations. This was important because an inability to change procedures is a commonly perceived barrier to using research from a nursing perspective. ${ }^{43}$

During REKAP, the two nurse champions developed clear objectives for BP management to be followed by the nursing staff. Modifications were made to existing BP monitoring flow sheets to provide visual cuing with regard to normal BP ranges for patients, including adjusted targets for diabetic patients. Clinical education using 30-minute in-services were provided in which staff received instruction on stroke rehabilitation guidelines, the new protocol, practical application, and case studies. For any staff unable to attend, the material was reviewed individually with one of the project champions. Reference material, such as standard doses and side effects, for antihypertensive medication in the hospital formulary was synthesized into a single page and placed within patient charts for easy accessibility.

\section{Monitor}

Monitoring allows for the provision of feedback regarding the extent to which a new strategy is incorporated into practice. ${ }^{8}$ On our stroke rehabilitation unit, once an intervention strategy was developed, it was trialed for a 6-week period. As necessary, the strategy was modified to accommodate the dynamic nature of the rehabilitation unit and the fluidity of the KTA cycle. During this trial period, random real-time audits were conducted to assess whether guideline adherence had improved. Sporadic on-unit audits were conducted by research staff using the bedside medical charts. This "just-in-time feedback" was used to identify issues of noncompliance and increase accountability. The results of these audits were presented to clinicians and management to maintain transparency and encourage open communication. The clinicians and management were then able to offer direct feedback that was timely, individualized, nonpunitive, and customizable. $^{44}$ This 6-week timeframe sufficiently allowed for the protocol to be trialed and modified based on feedback from staff. If additional modifications were necessary, the trial period was extended. Following the trial period, the official protocol was implemented.

\section{Evaluate}

The evaluation phase aims to determine if a change initiative has actually made an impact on outcomes. ${ }^{8}$ Successful outcomes can take the form of a change in attitude, behavior, and/or knowledge, ${ }^{45}$ or as a change at a structural, process, and outcome level. ${ }^{46}$ The primary outcome of REKAP was improvement in adherence to best practice recommendations for stroke rehabilitation; therefore, guideline adherence was reassessed using a second retrospective chart audit conducted approximately 6 months after the implementation of the each guideline adherence strategy.

In evaluating REKAP strategies, the second audit showed depression screening (originally $4.9 \%$ ) $^{32}$ increased to more than $40 \%$; because of a lack of baseline data assessing how many patients had a true diagnosis of depression, the evaluation of whether this protocol resulted in improved treatment is unknown. Benzodiazepine use decreased from $25 \%$ to $12.5 \%$. For hypertension management, the number of patients with unrecorded BP days was significantly lower in the follow-up audit $(\mathrm{p}<0.001)$ compared with the baseline audit (Table 1). The mean number of unrecorded BP days was also reduced significantly $(\mathrm{p}=0.013)$ from the initial audit $(4.9 \pm 4.7$ days) to the follow-up $(1.3 \pm 0.5$ days $)$. Other areas evaluated, such as bladder management, did not show significant improvements when evaluated, and require continued efforts to improve adherence rates. It appeared that strategies focused on a single discipline (e.g. nursing) were most successful.

\section{SUSTAIN}

The sustainability of the cycle is driven by continuous feedback from one stage of the cycle to another. ${ }^{8}$ As new barriers arise, new care gaps and new evidence is identified which requires constant modification of the intervention strategies. The need for frequent feedback is not only driven by external factors such as health care funding models and resource allocation, but also by internal factors such as stagnation in the motivation for change and the containment of change initiatives to specific units. ${ }^{47}$

REKAP embedded strategies for sustainability within each stage of the action cycle. All strategies were created collaboratively with staff members while keeping the local context in mind. Changes were made to staff orientation packages so that all new team members were aware of unit expectations. Existing staff members were involved by being educated and engaged

Table 1: Comparison of findings between the initial and follow-up audit

\begin{tabular}{l|c|c}
\hline & Initial audit $\mathbf{N}(\%)$ & Follow-up audit N $(\%)$ \\
\hline Monitoring & $\mathbf{n = 1 2 0}$ & $\mathbf{n = 1 1 8}$ \\
\hline 2 BP readings recorded per day, everyday & $0(0.0)$ & $31(26.3)$ \\
\hline$\geq 1$ unrecorded day (no BP recorded) & $107(89.2)$ & $11(9.3)$ \\
\hline Identified hypertension & & $<0.001$ \\
\hline$\geq 1$ hypertensive day & $88(73.3)$ & $102(86.4)$ \\
\hline Management of hypertensive patients & $\mathbf{n = 8 8}$ & $\mathbf{n = 1 0 2}$ \\
\hline Patients on antihypertensive medications & $78(88.6)$ & 0.015 \\
\hline$\geq 1$ untreated day & $46(52.3)$ & $95(93.1)$ \\
\hline$\geq 1$ medication change & $44(50.0)$ & $47(46.1)$ \\
\hline
\end{tabular}

$\mathrm{BP}=$ blood pressure. 
throughout the process to instill confidence in the process. Organizational culture is essential to sustainability, and when organizations provide continuous education, encourage collaborations, and incorporate guidelines into policies and procedures, adherence to evidence-based medicine is more likely to occur. ${ }^{42}$

\section{DisCUSSION}

The EBRSR uses a well-validated methodology and has proven to be critical infrastructure in Canada for ensuring an up-to-date, comprehensive evidence-based platform for continuing efforts toward moving best evidence into practice in stroke rehabilitation. REKAP used an audit-feedback method that followed the action cycle of the KTA process. It is a system for identifying, prioritizing, and guiding clinician behaviour and has successfully improved adherence to best practice recommendations on a single stroke rehabilitation unit in Southwestern Ontario, Canada. Through a retrospective audit, care gaps in many facets of stroke rehabilitation were identified by recommendations developed and promoted by the Canadian Best Practice Recommendations for Stroke Care. ${ }^{24}$ The application of this framework has significantly and positively influenced clinical practice and patient outcomes; its use is encouraged in other centres to facilitate an equivalent cycle of change. Although the strategies need to be individualized to the centre where they are being implemented, the overall KT framework can be easily used to evaluate the use of evidence-based medicine across other stroke units as well as in other populations.

Throughout this process, it has become clear that there must also be a concerted effort to ensure that guidelines themselves are both comprehensive and prescriptive. Recommendations that are nonspecific and vague contribute to lower compliance rates among staff members because they are too difficult to apply ${ }^{48}$ and challenging to measure. An additional consideration when evaluating adherence is whether the requirement is applicable and pragmatic to all patients. The discrepancy between standards of care and best practices may reflect differences between an "acceptable minimum standard" and "optimal care." this audit feedback process is not only promising in terms of a knowledge translation initiative but reciprocally, it is also a valuable exercise in identifying limitations in CPGs and the way research findings are translated into clinical practice.

\section{ACKNOWLEDGEMENT}

We would like to thank the Canadian Stroke Network, Heart and Stroke Foundation, as well as the Academic Medical Organization of Southwestern Ontario (AMOSO) funded through the Ontario Ministry of Health and Long-Term Care for their generous support.

\section{Disclosures}

The authors do not have anything to disclose. The authors have no conflicts of interest to declare.

\section{REFERENCES}

1. Public Health Agency Canada. Tracking Heart Disease and Stroke in Canada. Toronto, Ontario, Canada: Public Health Agency Canada; 2011.

2. Factors predicting discharge home from inpatient rehabilitation after stroke. Analysis in Brief. [Internet]. 2009. Available from: http:// secure.cihi.ca/cihiweb/dispPage.jsp?cw_page $=P_{-}$_2070_E\&cw_ topic $=2070 \&$ cw_rel=AR_2891_E.
3. Sackett DL, Rosenberg WM, Gray JA, Haynes RB, Richardson WS. Evidence based medicine: what it is and what it isn't. Clinical Orthop Relat Res. 1996;455:3-5.

4. McGlynn EA, Asch SM, Adams J, et al. The quality of health care delivered to adults in the United States. N Engl J Med. 2003; 348:2635-45.

5. Grol R. Successes and failures in the implementation of evidencebased guidelines for clinical practice. Med Care. 2001;39(8 Suppl 2): i46-54.

6. Green LW, Ottoson JM, Garcia C, Hiatt RA. Diffusion theory and knowledge dissemination, utilization, and integration in public health. Annu Rev Public Health. 2009;30:151-74.

7. Grimshaw JM, Eccles MP, Lavis JN, Hill SJ, Squires JE. Knowledge translation of research findings. Implement Sci. 2012;7:50.

8. Graham ID, Logan J, Harrison MB, et al. Lost in knowledge translation: time for a map? J Contin Educ Health Prof. 2006;26: 13-24.

9. Teasell R, Allen L, Hussein N. Evidence-based review of stroke rehabilitation. 16th ed. 2014 [cited 2014 May 7]. Available from: www.ebrsr.com.

10. Petzold A, Korner-Bitensky N, Menon A. Using the knowledge to action process model to incite clinical change. J Contin Educ Health Prof. 2010;30:167-71.

11. Hadely KA, Power E, O'Halloran R. Speech pathologists' experiences with stroke clinical practice guidelines and the barriers and facilitators influencing their use: a national descriptive study. BMC. 2014;14:110.

12. Glasziou P, Haynes B. The paths from research to improved health outcomes. Evid Based Nurs. 2005;8:36-8.

13. McIntyre A, Richardson M, Janzen S, Hussein N, Teasell R. The evolution of stroke rehabilitation randomized controlled trials. Int J Stroke. 2014;9:789-92.

14. Canadian Institutes of Health Research. Strategy for patient-oriented research: a discussion paper for a 10 -year plan to change health care using the levers of research. CIHR; 2010.

15. Drummond D. Commission on the Reform of Ontario's Public Services: Public sevices for Ontarians: A path to sustainability and excellence. Toronto: Queen's Printer for Ontario; 2012.

16. Tunis SR, Stryer DB, Clancy CM. Practical clinical trials: increasing the value of clinical research for decision making in clinical and health policy. JAMA. 2003;290:1624-32.

17. Clarke M, Alderson P, Chalmers I. Discussion sections in reports of controlled trials published in general medical journals. JAMA. 2002;287:2799-801.

18. Sherrington C, Herbert RD, Maher CG, Moseley AM. PEDro. A database of randomized trials and systematic reviews in physiotherapy. Man Ther. 2000;5:223-6.

19. Jadad AR, Cook DJ, Jones A, et al. Methodology and reports of systematic reviews and meta-analyses: a comparison of Cochrane reviews with articles published in paper-based journals. JAMA. 1998;280:278-80.

20. Teasell R, Foley N, Salter K, Meyer M, McClure A, Graham R. Impact of the Stroke Rehabilitation Evidence-Based Review. J Rehabil Med. 2010;42:1004-5.

21. Teasell R, Foley N, Bhogal S, Bagg S, Jutai J. Evidence-based practice and setting basic standards for stroke rehabilitation in Canada. Top Stroke Rehabil. 2006;13:59-65.

22. Bayley MT, Hurdowar A, Teasell R, et al. Priorities for stroke rehabilitation and research: results of a 2003 Canadian Stroke Network consensus conference. Arch Phys Med Rehabil. 2007; 88:526-8.

23. Miller EL, Murray L, Richards L, et al. Comprehensive overview of nursing and interdisciplinary rehabilitation care of the stroke patient: a scientific statement from the American Heart Association. Stroke. 2010;41:2402-48.

24. Lindsay P, Gubitz G, Bayley M, et al. Canadian Best Practice Recommendations for Stroke Care (Update 2010). Ottawa, Ontario Canada: Canadian Stroke Network; 2010.

25. National Stroke Foundation. Clinical Guidelines for Stroke Management 2010. Melbourne, Australia; 2010.

26. Duncan PW, Horner RD, Reker DM, et al. Adherence to postacute rehabilitation guidelines is associated with functional recovery in stroke. Stroke. 2002;33:167-77. 
27. Nieuwlaat R, Olsson SB, Lip GY, et al. Guideline-adherent antithrombotic treatment is associated with improved outcomes compared with undertreatment in high-risk patients with atrial fibrillation. The Euro Heart Survey on Atrial Fibrillation. Am Heart J. 2007;153:1006-12.

28. Schwamm LH, Fonarow GC, Reeves MJ, et al. Get With the Guidelines-Stroke is associated with sustained improvement in care for patients hospitalized with acute stroke or transient ischemic attack. Circulation. 2009;119:107-15.

29. Menon A, Korner-Bitensky N, Kastner M, McKibbon KA, Straus S. Strategies for rehabilitation professionals to move evidence-based knowledge into practice: a systematic review. J Rehabil Med. 2009;41:1024-32.

30. Graham ID, Beardall S, Carter AO, Tetroe J, Davies B. The state of the science and art of practice guidelines development, dissemination and evaluation in Canada. J Eval Clin Pract. 2003;9:195-202.

31. Abilleira S, Gallofre M, Ribera A, Sanchez E, Tresserras R. Quality of in-hospital stroke care according to evidence-based performance measures: results from the first audit of stroke, Catalonia, Spain. Stroke. 2009;40:1433-8.

32. Salter K, McClure A, Mahon H, Foley N, Teasell R. Adherence to Canadian best practice recommendations for stroke care: assessment and management of poststroke depression in an Ontario rehabilitation facility. Topics in Stroke Rehabilitation. 2012;19:132-40.

33. McClure JA, Salter K, Foley N, Mahon H, Teasell R. Adherence to Canadian Best Practice Recommendations for Stroke Care: vascular cognitive impairment screening and assessment practices in an Ontario inpatient stroke rehabilitation facility. Top Stroke Rehabil. 2012;19:141-8.

34. Foley N, Salter K, Teasell R, Meyer M, McClure A, Britt E. A survey of scheduled therapy time for assessment and treatment of patients admitted for inpatient stroke rehabilitation. Stroke. 2010;41:e506.

35. Cassidy C, Leci E, Meyer M, Janzen S, Teasell R. Adherence to Canadian guidelines for benzodiazepines on a stroke rehabilitation unit. J Rehabil Med. 2012;44:1002.

36. Chew S, Armstrong N, Martin G. Institutionalising knowledge brokering as a sustainable knowledge translation solution in healthcare: How can it work in practice? Evid Policy. 2013;9:335-51.

37. Cabana MD, Rand CS, Powe NR, et al. Why don't physicians follow clinical practice guidelines? A framework for improvement. JAMA. 1999;282:1458-65.
38. Bayley MT, Hurdowar A, Richards CL, et al. Barriers to implementation of stroke rehabilitation evidence: findings from a multi-site pilot project. Disabil Rehabil. 2012;34:1633-8.

39. Gache K, Leleu H, Nitenberg G, Woimant F, Ferrua M, Minvielle E. Main barriers to effective implementation of stroke care pathways in France: a qualitative study. BMC Health Serv Res. 2014; $14: 95$

40. McCluskey A, Vratsistas-Curto A, Schurr K. Barriers and enablers to implementing multiple stroke guideline recommendations: a qualitative study. BMC Health Serv Res. 2013;13:323.

41. Prior M, Guerin M, Grimmer-Somers K. The effectiveness of clinical guideline implementation strategies-a synthesis of systematic review findings. J Eval Clin Pract. 2008;14:888-97.

42. Davis D. Continuing education, guideline implementation, and the emerging transdisciplinary field of knowledge translation. J Contin Educ Health Prof. 2006;26:5-12.

43. Kajermo KN, Bostrom AM, Thompson DS, Hutchinson AM, Estabrooks CA, Wallin L. The BARRIERS scale - the barriers to research utilization scale: a systematic review. Implement Sci. 2010;5:32.

44. Hysong SJ, Best RG, Pugh JA. Audit and feedback and clinical practice guideline adherence: making feedback actionable. Implement Sci. 2006;1:9.

45. Woolf SH. Practice guidelines: a new reality in medicine. III. Impact on patient care. Arch Intern Med. 1993;153:2646-55.

46. Straus S, Tetroe J, Graham ID, Zwarenstein M, Bhattacheryya O. Monitoring knowledge use and evaluating outcomes of knowledge use 2011 [June 17, 2014]. Available from: http://www.cihr-irsc. gc.ca/e/40618.html\#toc.

47. National Institute for Health and Clinical Excellence. How to change practice: understand, identify and overcome barriers to change. London, UK: National Institute for Health and Clinical Excellence; 2007.

48. Grol R, Dalhuijsen J, Thomas S, Veld C, Rutten G, Mokkink H. Attributes of clinical guidelines that influence use of guidelines in general practice: observational study. BMJ. 1998;317: 858-61.

49. Groah SL, Libin A, Lauderdale M, Kroll T, DeJong G, Hsieh J. Beyond the evidence-based practice paradigm to achieve best practice in rehabilitation medicine: a clinical review. PMR. 2009; 1:941-50. 